\title{
Search for a Stable Six-Quark State at BABAR
}

J. P. Lees, ${ }^{1}$ V. Poireau,,${ }^{1}$ V. Tisserand, ${ }^{1}$ E. Grauges,${ }^{2}$ A. Palano, ${ }^{3}$ G. Eigen, ${ }^{4}$ D. N. Brown, ${ }^{5}$ Yu. G. Kolomensky, ${ }^{5}$ M. Fritsch, ${ }^{6}$ H. Koch, ${ }^{6}$ T. Schroeder, ${ }^{6}$ C. Hearty, ${ }^{7 a}, 7 \mathrm{~b}$ T. S. Mattison, ${ }^{7 \mathrm{~b}}$ J. A. McKenna, ${ }^{7 \mathrm{~b}}$ R. Y. So, ${ }^{7 \mathrm{~b}}$ V. E. Blinov, ${ }^{8 \mathrm{a}, 8 \mathrm{~b}, 8 \mathrm{c}}$ A. R. Buzykaev, ${ }^{8 \mathrm{a}}$ V. P. Druzhinin, ${ }^{8 a, 8 b}$ V. B. Golubev, ${ }^{8 a, 8 b}$ E. A. Kozyrev, ${ }^{8 a, 8 b}$ E. A. Kravchenko, ${ }^{8 a, 8 b}$ A. P. Onuchin, ${ }^{8 a, 8 b, 8 c}$

S. I. Serednyakov, ${ }^{8 \mathrm{a}, 8 \mathrm{~b}}$ Yu. I. Skovpen,${ }^{8 \mathrm{a}, 8 \mathrm{~b}}$ E. P. Solodov, ${ }^{8 \mathrm{a}, 8 \mathrm{~b}}$ K. Yu. Todyshev,${ }^{8 \mathrm{a}, 8 \mathrm{~b}}$ A. J. Lankford, ${ }^{9}$ J. W. Gary, ${ }^{10}$ O. Long, ${ }^{10}$ A. M. Eisner, ${ }^{11}$ W. S. Lockman, ${ }^{11}$ W. Panduro Vazquez, ${ }^{11}$ D. S. Chao, ${ }^{12}$ C. H. Cheng, ${ }^{12}$ B. Echenard, ${ }^{12}$ K. T. Flood, ${ }^{12}$ D. G. Hitlin, ${ }^{12}$ J. Kim, ${ }^{12}$ Y. Li, ${ }^{12}$ T. S. Miyashita, ${ }^{12}$ P. Ongmongkolkul, ${ }^{12}$ F. C. Porter, ${ }^{12}$ M. Röhrken, ${ }^{12}$ Z. Huard ${ }^{13}$

B. T. Meadows, ${ }^{13}$ B. G. Pushpawela, ${ }^{13}$ M. D. Sokoloff, ${ }^{13}$ L. Sun, ${ }^{13, \dagger}$ J. G. Smith, ${ }^{14}$ S. R. Wagner, ${ }^{14}$ D. Bernard, ${ }^{15}$

M. Verderi, ${ }^{15}$ D. Bettoni ${ }^{16 a}$ C. Bozzi, ${ }^{16 a}$ R. Calabrese, ${ }^{16 a, 16 b}$ G. Cibinetto, ${ }^{16 a, 16 b}$ E. Fioravanti, ${ }^{16 a, 16 b}$ I. Garzia, ${ }^{16 a, 16 b}$ E. Luppi, ${ }^{16 a, 16 b}$ V. Santoro, ${ }^{16 a}$ A. Calcaterra,${ }^{17}$ R. de Sangro, ${ }^{17}$ G. Finocchiaro, ${ }^{17}$ S. Martellotti, ${ }^{17}$ P. Patteri, ${ }^{17}$ I. M. Peruzzi ${ }^{17}$ M. Piccolo,${ }^{17}$ M. Rotondo, ${ }^{17}$ A. Zallo, ${ }^{17}$ S. Passaggio, ${ }^{18}$ C. Patrignani, ${ }^{18,}$ H. M. Lacker, ${ }^{19}$ B. Bhuyan, ${ }^{20}$ U. Mallik, ${ }^{21}$ C. Chen, ${ }^{22}$ J. Cochran, ${ }^{22}$ S. Prell, ${ }^{22}$ A. V. Gritsan, ${ }^{23}$ N. Arnaud, ${ }^{24}$ M. Davier, ${ }^{24}$ F. Le Diberder, ${ }^{24}$ A. M. Lutz ${ }^{24}$ G. Wormser,${ }^{24}$ D. J. Lange, ${ }^{25}$ D. M. Wright, ${ }^{25}$ J. P. Coleman, ${ }^{26}$ E. Gabathuler, ${ }^{26,}{ }^{*}$ D. E. Hutchcroft, ${ }^{26}$ D. J. Payne, ${ }^{26}$ C. Touramanis, ${ }^{26}$ A. J. Bevan, ${ }^{27}$ F. Di Lodovico, ${ }^{27}$ R. Sacco, ${ }^{27}$ G. Cowan, ${ }^{28}$ Sw. Banerjee, ${ }^{29}$ D. N. Brown, ${ }^{29}$ C. L. Davis, ${ }^{29}$ A. G. Denig, ${ }^{30}$ W. Gradl, ${ }^{30}$ K. Griessinger, ${ }^{30}$ A. Hafner, ${ }^{30}$ K. R. Schubert, ${ }^{30}$ R. J. Barlow, ${ }^{31,}$ G. D. Lafferty, ${ }^{31}$ R. Cenci, ${ }^{32}$ A. Jawahery, ${ }^{32}$ D. A. Roberts, ${ }^{32}$ R. Cowan, ${ }^{33}$ S. H. Robertson, ${ }^{34 a, 34 b}$ R. M. Seddon, ${ }^{34 b}$ B. Dey, ${ }^{35 a}$ N. Neri, ${ }^{35 a}$ F. Palombo, ${ }^{35 a}, 35 b$ R. Cheaib, ${ }^{36}$ L. Cremaldi, ${ }^{36}$ R. Godang, ${ }^{36,9}$ D. J. Summers, ${ }^{36}$ P. Taras, ${ }^{37}$ G. De Nardo, ${ }^{38}$ C. Sciacca, ${ }^{38}$ G. Raven, ${ }^{39}$ C. P. Jessop ${ }^{40}$ J. M. LoSecco, ${ }^{40}$ K. Honscheid, ${ }^{41}$ R. Kass,${ }^{41}$ A. Gaz, ${ }^{42 a}$ M. Margoni, ${ }^{42 a, 42 b}$ M. Posocco, ${ }^{42 a}$ G. Simi, ${ }^{42 a, 42 b}$ F. Simonetto, ${ }^{42 a, 42 b}$ R. Stroili, ${ }^{42 a, 42 b}$ S. Akar, ${ }^{43}$ E. Ben-Haim, ${ }^{43}$ M. Bomben, ${ }^{43}$ G. R. Bonneaud, ${ }^{43}$ G. Calderini, ${ }^{43}$ J. Chauveau, ${ }^{43}$ G. Marchiori, ${ }^{43}$ J. Ocariz, ${ }^{43}$ M. Biasini, ${ }^{44,44 b}$ E. Manoni, ${ }^{44 a}$ A. Rossi, ${ }^{44 a}$ G. Batignani, ${ }^{45 a, 45 b}$ S. Bettarini, ${ }^{45 a, 45 b}$ M. Carpinelli, ${ }^{45 a, 45 b, * *}$ G. Casarosa, ${ }^{45 a, 45 b}$ M. Chrzaszcz, ${ }^{45 a}$ F. Forti, ${ }^{45 a, 45 b}$ M. A. Giorgi, ${ }^{45 a, 45 b}$ A. Lusiani, ${ }^{45 a, 45 c}$ B. Oberhof, ${ }^{45 a, 45 b}$ E. Paoloni, ${ }^{45 a, 45 b}$ M. Rama, ${ }^{45 a}$ G. Rizzo, ${ }^{45 a, 45 b}$ J. J. Walsh, ${ }^{45 a}$ L. Zani, ${ }^{45,45 b}$ A. J. S. Smith ${ }^{46}$ F. Anulli, ${ }^{47 a}$ R. Faccini, ${ }^{47 a, 47 b}$ F. Ferrarotto, ${ }^{47 a}$

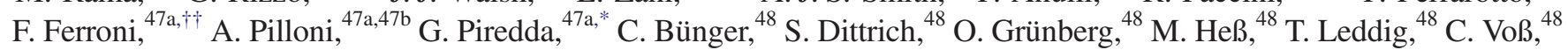
R. Waldi, ${ }^{48}$ T. Adye, ${ }^{49}$ F. F. Wilson, ${ }^{49}$ S. Emery, ${ }^{50}$ G. Vasseur, ${ }^{50}$ D. Aston, ${ }^{51}$ C. Cartaro,${ }^{51}$ M. R. Convery, ${ }^{51}$ J. Dorfan, ${ }^{51}$ W. Dunwoodie, ${ }^{51}$ M. Ebert, ${ }^{51}$ R. C. Field, ${ }^{51}$ B. G. Fulsom, ${ }^{51}$ M. T. Graham,${ }^{51}$ C. Hast, ${ }^{51}$ W. R. Innes,${ }^{51, *}$ P. Kim ${ }^{51}$ D. W. G. S. Leith, ${ }^{51}$ S. Luitz, ${ }^{51}$ D. B. MacFarlane, ${ }^{51}$ D. R. Muller, ${ }^{51}$ H. Neal,${ }^{51}$ B. N. Ratcliff, ${ }^{51}$ A. Roodman, ${ }^{51}$ M. K. Sullivan, ${ }^{51}$ J. Va'vra, ${ }^{51}$ W. J. Wisniewski, ${ }^{51}$ M. V. Purohit, ${ }^{52}$ J. R. Wilson, ${ }^{52}$ A. Randle-Conde, ${ }^{53}$ S. J. Sekula, ${ }^{53}$ H. Ahmed, ${ }^{54}$ M. Bellis, ${ }^{55}$ P. R. Burchat,${ }^{55}$ E. M. T. Puccio, ${ }^{55}$ M. S. Alam,${ }^{56}$ J. A. Ernst, ${ }^{56}$ R. Gorodeisky, ${ }^{57}$ N. Guttman,${ }^{57}$ D. R. Peimer, ${ }^{57}$ A. Soffer, ${ }^{57}$ S. M. Spanier, ${ }^{58}$ J. L. Ritchie, ${ }^{59}$ R. F. Schwitters ${ }^{59}$ J. M. Izen, ${ }^{60}$ X. C. Lou, ${ }^{60}$ F. Bianchi, ${ }^{61 a, 61 b}$ F. De Mori, ${ }^{61 \mathrm{a}, 61 \mathrm{~b}}$ A. Filippi, ${ }^{61 \mathrm{a}}$ D. Gamba,${ }^{61 \mathrm{a}, 61 \mathrm{~b}}$ L. Lanceri, ${ }^{62}$ L. Vitale,${ }^{62}$ F. Martinez-Vidal, ${ }^{63}$ A. Oyanguren, ${ }^{63}$ J. Albert, ${ }^{64 \mathrm{~b}}$ A. Beaulieu, ${ }^{64 \mathrm{~b}}$ F. U. Bernlochner, ${ }^{64 \mathrm{~b}}$ G. J. King, ${ }^{64 \mathrm{~b}}$ R. Kowalewski, ${ }^{64 \mathrm{~b}}$ T. Lueck, ${ }^{64 \mathrm{~b}}$ I. M. Nugent, ${ }^{64 \mathrm{~b}}$ J. M. Roney ${ }^{64 \mathrm{~b}}$ R. J. Sobie, ${ }^{64 a, 64 b}$ N. Tasneem, ${ }^{64 b}$ T. J. Gershon, ${ }^{65}$ P. F. Harrison, ${ }^{65}$ T. E. Latham, ${ }^{65}$ R. Prepost, ${ }^{66}$ and S. L. Wu ${ }^{66}$

\section{(BABAR Collaboration)}

\author{
${ }^{1}$ Laboratoire d'Annecy-le-Vieux de Physique des Particules (LAPP), Université de Savoie, \\ CNRS/IN2P3, F-74941 Annecy-Le-Vieux, France \\ ${ }^{2}$ Universitat de Barcelona, Facultat de Fisica, Departament ECM, E-08028 Barcelona, Spain \\ ${ }^{3}$ INFN Sezione di Bari and Dipartimento di Fisica, Università di Bari, I-70126 Bari, Italy \\ ${ }^{4}$ University of Bergen, Institute of Physics, N-5007 Bergen, Norway \\ ${ }^{5}$ Lawrence Berkeley National Laboratory and University of California, Berkeley, California 94720, USA \\ ${ }^{6}$ Ruhr Universität Bochum, Institut für Experimentalphysik 1, D-44780 Bochum, Germany \\ ${ }^{7 a}$ Institute of Particle Physics, Vancouver, British Columbia, Canada V6T IZ1 \\ ${ }^{7 b}$ University of British Columbia, Vancouver, British Columbia, Canada V6T $1 Z 1$ \\ ${ }^{8 a}$ Budker Institute of Nuclear Physics SB RAS, Novosibirsk 630090, Russia \\ ${ }^{8 b}$ Novosibirsk State University, Novosibirsk 630090, Russia \\ ${ }^{8 \mathrm{c} N o v o s i b i r s k}$ State Technical University, Novosibirsk 630092, Russia \\ ${ }^{9}$ University of California at Irvine, Irvine, California 92697, USA \\ ${ }^{10}$ University of California at Riverside, Riverside, California 92521, USA \\ ${ }^{11}$ University of California at Santa Cruz, Institute for Particle Physics, Santa Cruz, California 95064, USA \\ ${ }^{12}$ California Institute of Technology, Pasadena, California 91125, USA
}


${ }^{13}$ University of Cincinnati, Cincinnati, Ohio 45221, USA

${ }^{14}$ University of Colorado, Boulder, Colorado 80309, USA

${ }^{15}$ Laboratoire Leprince-Ringuet, Ecole Polytechnique, CNRS/IN2P3, F-91128 Palaiseau, France

${ }^{16 a}$ INFN Sezione di Ferrara, I-44122 Ferrara, Italy

${ }^{16 \mathrm{~b}}$ Dipartimento di Fisica e Scienze della Terra, Università di Ferrara, I-44122 Ferrara, Italy

${ }^{17}$ INFN Laboratori Nazionali di Frascati, I-00044 Frascati, Italy

${ }^{18}$ INFN Sezione di Genova, I-16146 Genova, Italy

${ }^{19}$ Humboldt-Universität zu Berlin, Institut für Physik, D-12489 Berlin, Germany

${ }^{20}$ Indian Institute of Technology Guwahati, Guwahati, Assam, 781 039, India

${ }^{21}$ University of Iowa, Iowa City, Iowa 52242, USA

${ }^{22}$ Iowa State University, Ames, Iowa 50011, USA

${ }^{23}$ Johns Hopkins University, Baltimore, Maryland 21218, USA

${ }^{24}$ Laboratoire de l'Accélérateur Linéaire, IN2P3/CNRS et Université Paris-Sud 11, Centre Scientifique d'Orsay, F-91898 Orsay Cedex, France

${ }^{25}$ Lawrence Livermore National Laboratory, Livermore, California 94550, USA

${ }^{26}$ University of Liverpool, Liverpool L69 7ZE, United Kingdom

${ }^{27}$ Queen Mary, University of London, London E1 4NS, United Kingdom

${ }^{28}$ University of London, Royal Holloway and Bedford New College, Egham, Surrey TW20 OEX, United Kingdom

${ }^{29}$ University of Louisville, Louisville, Kentucky 40292, USA

${ }^{30}$ Johannes Gutenberg-Universität Mainz, Institut für Kernphysik, D-55099 Mainz, Germany

${ }^{31}$ University of Manchester, Manchester M13 9PL, United Kingdom

${ }^{32}$ University of Maryland, College Park, Maryland 20742, USA

${ }^{33}$ Massachusetts Institute of Technology, Laboratory for Nuclear Science, Cambridge, Massachusetts 02139, USA

${ }^{34 a}$ Institute of Particle Physics, Montréal, Québec, Canada H3A 2T8

${ }^{34 \mathrm{~b}}$ McGill University, Montréal, Québec, Canada H3A 278

${ }^{35 a}$ INFN Sezione di Milano, I-20133 Milano, Italy

${ }^{35 \mathrm{~b}}$ Dipartimento di Fisica, Università di Milano, I-20133 Milano, Italy

${ }^{36}$ University of Mississippi, University, Mississippi 38677, USA

${ }^{37}$ Université de Montréal, Physique des Particules, Montréal, Québec, Canada H3C 3J7

${ }^{38}$ INFN Sezione di Napoli and Dipartimento di Scienze Fisiche, Università di Napoli Federico II, I-80126 Napoli, Italy

${ }^{39}$ NIKHEF, National Institute for Nuclear Physics and High Energy Physics, NL-1009 DB Amsterdam, Netherlands

${ }^{40}$ University of Notre Dame, Notre Dame, Indiana 46556, USA

${ }^{41}$ Ohio State University, Columbus, Ohio 43210, USA

${ }^{42 a}$ INFN Sezione di Padova, I-35131 Padova, Italy

${ }^{42 \mathrm{~b}}$ Dipartimento di Fisica, Università di Padova, I-35131 Padova, Italy

${ }^{43}$ Laboratoire de Physique Nucléaire et de Hautes Energies, IN2P3/CNRS, Université Pierre et Marie Curie-Paris6, Université Denis Diderot-Paris7, F-75252 Paris, France

${ }^{44 a}$ INFN Sezione di Perugia, I-06123 Perugia, Italy

${ }^{44 \mathrm{~b}}$ Dipartimento di Fisica, Università di Perugia, I-06123 Perugia, Italy

${ }^{45 a}$ INFN Sezione di Pisa, I-56127 Pisa, Italy

${ }^{45 \mathrm{~b}}$ Dipartimento di Fisica, Università di Pisa, I-56127 Pisa, Italy

${ }^{45 c}$ Scuola Normale Superiore di Pisa, I-56127 Pisa, Italy

${ }^{46}$ Princeton University, Princeton, New Jersey 08544, USA

${ }^{47 a}$ INFN Sezione di Roma, I-00185 Roma, Italy

${ }^{47 \mathrm{~b}}$ Dipartimento di Fisica, Università di Roma La Sapienza, I-00185 Roma, Italy

${ }^{48}$ Universität Rostock, D-18051 Rostock, Germany

${ }^{49}$ Rutherford Appleton Laboratory, Chilton, Didcot, Oxon, OX11 OQX, United Kingdom

${ }^{50}$ CEA, Irfu, SPP, Centre de Saclay, F-91191 Gif-sur-Yvette, France

${ }^{51}$ SLAC National Accelerator Laboratory, Stanford, California 94309 USA

${ }^{52}$ University of South Carolina, Columbia, South Carolina 29208, USA

${ }^{53}$ Southern Methodist University, Dallas, Texas 75275, USA

${ }^{54}$ St. Francis Xavier University, Antigonish, Nova Scotia, Canada B2G 2W5

${ }^{55}$ Stanford University, Stanford, California 94305, USA

${ }^{56}$ State University of New York, Albany, New York 12222, USA

${ }^{57}$ Tel Aviv University, School of Physics and Astronomy, Tel Aviv, 69978, Israel

${ }^{58}$ University of Tennessee, Knoxville, Tennessee 37996, USA

${ }^{59}$ University of Texas at Austin, Austin, Texas 78712, USA

${ }^{60}$ University of Texas at Dallas, Richardson, Texas 75083, USA

${ }^{61 \mathrm{a}}$ INFN Sezione di Torino, I-10125 Torino, Italy

${ }^{61 \mathrm{~b}}$ Dipartimento di Fisica, Università di Torino, I-10125 Torino, Italy 
${ }^{62}$ INFN Sezione di Trieste and Dipartimento di Fisica, Università di Trieste, I-34127 Trieste, Italy

${ }^{63}$ IFIC, Universitat de Valencia-CSIC, E-46071 Valencia, Spain

${ }^{64 a}$ Institute of Particle Physics, Victoria, British Columbia, Canada V8W $3 P 6$

${ }^{64 \mathrm{~b}}$ University of Victoria, Victoria, British Columbia, Canada V8W $3 P 6$

${ }^{65}$ Department of Physics, University of Warwick, Coventry CV4 7AL, United Kingdom

${ }^{66}$ University of Wisconsin, Madison, Wisconsin 53706, USA

(Received 14 November 2018; revised manuscript received 24 January 2019; published 22 February 2019)

Recent investigations have suggested that the six-quark combination uuddss could be a deeply bound state $(S)$ that has eluded detection so far, and a potential dark matter candidate. We report the first search for a stable, doubly strange six-quark state in $\Upsilon \rightarrow S \bar{\Lambda} \bar{\Lambda}$ decays based on a sample of $90 \times 10^{6} \Upsilon(2 S)$ and $110 \times 10^{6} \Upsilon(3 S)$ decays collected by the $B A B A R$ experiment. No signal is observed, and $90 \%$ confidence level limits on the combined $\Upsilon(2 S, 3 S) \rightarrow S \bar{\Lambda} \bar{\Lambda}$ branching fraction in the range $(1.2-1.4) \times 10^{-7}$ are derived for $m_{S}<2.05 \mathrm{GeV}$. These bounds set stringent limits on the existence of such exotic particles.

DOI: 10.1103/PhysRevLett.122.072002

A new stable state of matter may still be undiscovered. While the vast majority of known hadrons can be described as either quark-antiquark or three-quark combinations, other multiquark possibilities are allowed by quantum chromodynamics (QCD). Among those, the six-quark configuration uuddss is of particular interest, as its spatial wave function is completely symmetric, and generic arguments imply that it should be the most tightly bound sixquark state (see, e.g., Ref. [1]). This property was already noticed by Jaffe 40 years ago [2]. He predicted the existence of a loosely bound uuddss state with a mass close to $2150 \mathrm{MeV}$ [3], dubbed the H-dibaryon. As its mass is above the $m_{p}+m_{e}+m_{\Lambda}=2055 \mathrm{MeV}$ threshold, the $\mathrm{H}$-dibaryon would have a typical weak interaction lifetime. Numerous negative experimental results were taken as evidence against such a particle, including observations of doubly strange hypernuclei decays [4,5], searches for narrow $\Lambda p \pi^{-}$resonances in $\Upsilon$ decays [6], and direct searches for new neutral particles (see, e.g., Refs. [7-11]).

The situation is markedly different if the potential is deeply attractive, as advocated by Farrar [12]. Below $2055 \mathrm{MeV}$, the uuddss configuration acquires a cosmological lifetime, as its decay would have to proceed via doubly weak interactions, and it is absolutely stable if it is lighter than $2\left(m_{p}+m_{e}\right)=1878 \mathrm{MeV}$. Such a bound state, tentatively named $S$ [13], hasn't been excluded so far by hypernuclei decays or direct searches for long-lived neutral states (the latter were limited to masses above $\sim 2 \mathrm{GeV}$ due to the large neutron background [11]). While current lattice QCD calculations suggest a small value of the uuddss binding energy, at the level of $\mathcal{O}(10) \mathrm{MeV}$ [14,15], lattice

Published by the American Physical Society under the terms of the Creative Commons Attribution 4.0 International license. Further distribution of this work must maintain attribution to the author(s) and the published article's title, journal citation, and DOI. Funded by SCOAP. systematic uncertainties remain too large to rule out a deeply bound state.

Although not all authors agree (see, e.g., Refs. [16-18]), a stable six-quark state might also have cosmological implications. If dark matter is composed of nearly equal numbers of $u, d$, and $s$ quarks, its formation rate is driven by the quark-gluon plasma transition to the hadronic phase and the quark and antiquark abundances. As the same source is also responsible for determining the residual amount of ordinary matter in the universe, this framework would explain both the dark matter density and the baryon asymmetry, two seemingly unrelated quantities. A specific realization of this scenario, six-quark dark matter with $m_{S} \sim 1860-1880 \mathrm{MeV}$, can reproduce the observed ratio of dark matter to ordinary matter densities within 15\% [19].

Being a flavor singlet, the $S$ particle does not couple to pions or other mesons. The $S$-nucleon interaction cross section is expected to be suppressed compared to that of nucleon-nucleon interactions, and its production rate is several orders of magnitude below that for neutrons. Given that a low-mass $S$ is difficult to kinematically distinguish from a neutron, these attributes might explain why this state has escaped detection so far. Despite these difficulties, several search strategies have been proposed. Among them, the exclusive decay $\Upsilon \rightarrow S \bar{\Lambda} \bar{\Lambda}$ [20] stands out for its simplicity and robustness. The short-distance nature of the gluonic source increases the overlap with the compact $S$ wave function, enhancing its production rate compared to other mechanisms involving baryons. Heuristic arguments suggest an inclusive six-quark production rate in $\Upsilon(1 S, 2 S$, $3 S$ ) decays at the level of $10^{-7}$, albeit with significant uncertainties [12]. No specific prediction for the exclusive $S \bar{\Lambda} \bar{\Lambda}$ final state has been made so far, though this channel could conceivably account for a large fraction of the total production rate.

We report herein the first search for a stable, doubly strange six-quark configuration produced in $\Upsilon(2 S, 3 S)$ 
decays [21]. For completeness, we probe the entire mass range compatible with a stable state: $0 \mathrm{GeV}<m_{S}<$ $2.05 \mathrm{GeV}$. The analysis is based on a sample containing $90 \times 10^{6} \Upsilon(2 S)$ and $110 \times 10^{6} \Upsilon(3 S)$ decays collected with the BABAR detector at the PEP-II2 asymmetric-energy $e^{+} e^{-}$collider operated at the SLAC National Accelerator Laboratory. The integrated luminosities of the $\Upsilon(2 S)$ and $\Upsilon(3 S)$ samples are $14 \mathrm{fb}^{-1}$ and $28 \mathrm{fb}^{-1}$, respectively [22]. Additional samples of $428 \mathrm{fb}^{-1}$ collected at the $\Upsilon(4 S)$ peak, as well as in the vicinity of the $\Upsilon(2 S, 3 S)$ resonances, are used to estimate the background. The BABAR detector is described in detail elsewhere [23,24]. To avoid experimental bias, we examine the data signal region only after finalizing the analysis strategy.

Simulated events are used to optimize the selection procedure and assess the signal efficiency. Signal events are generated for $0 \mathrm{GeV}<m_{S}<2.2 \mathrm{GeV}$ in steps of $0.2 \mathrm{GeV}$. The $S$ angular distribution is simulated using an effective Lagrangian based on a constant matrix element for the different arrangements of angular momentum between the final state particles, assuming that angular momentum suppression effects are small [25] (see the Appendix for a detailed description). A second model based on a phase space distribution is used to assess systematic uncertainties. The interaction between six-quark states and matter is expected to be similar to that of neutrons, albeit with reduced cross sections. For the purpose of simulating the signal, we model these interactions similarly to those of neutrons. As an extreme alternative, we simulate six-quark states as noninteracting particles, and we assign the difference between these two models as a systematic uncertainty. To study the background, we generate generic $\Upsilon(2 S, 3 S, 4 S)$ decays with EVTGEN [26], while the continuum $e^{+} e^{-} \rightarrow q \bar{q}$ $(q=u, d, s, c)$ background is estimated using a datadriven approach described below. The detector acceptance and reconstruction efficiencies are determined using a Monte Carlo (MC) simulation based on GEANT4 [27]. Time-dependent detector inefficiencies and background conditions, as monitored during data-taking periods, are included in the simulation.

We select events containing at most five tracks and two $\Lambda$ candidates with the same strangeness, reconstructed in the $\Lambda \Lambda \rightarrow p \pi^{-} p \pi^{-}$final state with $1.10 \mathrm{GeV}<m_{p \pi}<$ $1.14 \mathrm{GeV}$. One additional track not associated with a $\Lambda$ candidate with a distance of closest approach from the primary interaction point (DOCA) larger than $5 \mathrm{~cm}$ is allowed to account for particles produced from secondary interactions with the detector material. The (anti)protons must be selected by particle identification (PID) algorithms. This requirement, which is approximately $95 \%$ efficient for identifying both protons and antiprotons, removes a large amount of background from four-pion final states. To further improve the signal purity, the $\Lambda$ flight vector is measured as the distance between the primary interaction point and the $\Lambda$ decay vertex. The flight significance of each $\Lambda$ candidate, defined as the length of this vector dived by its uncertainty, must be larger than 5 . The cosine of the angle between the $\Lambda$ momentum and the flight vector must also be greater than 0.9 . In addition, the total energy of clusters in the electromagnetic calorimeter not associated with charged particles, $E_{\text {extra }}$, must be less than $0.5 \mathrm{GeV}$. To account for possible interactions between the $S$ candidate and the calorimeter, the sum excludes clusters that are closer than an angle of $0.5 \mathrm{rad}$ to the inferred $S$ direction. Moreover, the distance between the cluster and the proton is required to be greater than $40 \mathrm{~cm}$ to reduce the contribution of cluster fragments. The $E_{\text {extra }}$ distribution after applying all other selection criteria is shown in Fig. 1. The background is dominated by hadronic events containing several strange baryons and additional charged and neutral particles, a fraction of which escapes undetected. The selection procedure is tuned to maximize the signal sensitivity, taking into account the systematic uncertainties related to $S$ production and interaction with detector material in the calculation. The $p \pi^{-}$mass distribution obtained after applying these criteria is shown in Fig. 2. A total of $8 \Upsilon \rightarrow S \bar{\Lambda} \bar{\Lambda}$ candidates are selected.

The events are then fit, imposing a mass constraint to each $\Lambda$ candidate and requiring a common origin, compatible with the beam interaction point within its uncertainty. We select combinations with $\chi^{2}<25$ (for 8 d.o.f.), retaining half of the previously selected candidates. The signal is identified as a peak in the recoil mass squared against the $\Lambda \Lambda$ system, $m_{\text {rec }}^{2}$, in the region $0 \mathrm{GeV}^{2} \lesssim m_{\text {rec }}^{2} \lesssim$ $5 \mathrm{GeV}^{2}$. The recoil mass squared allows for negative values arising from the limited resolution on the reconstructed $\Lambda$

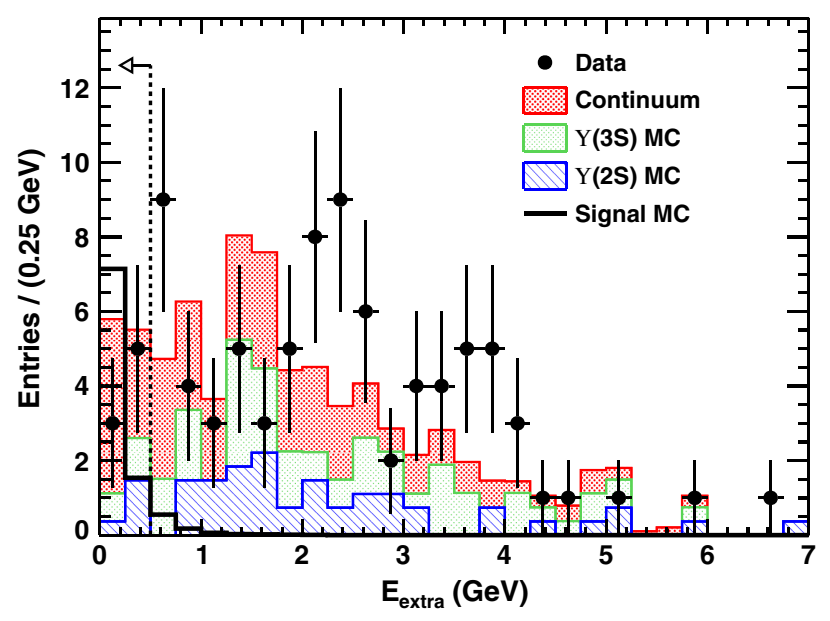

FIG. 1. The distribution of the extra neutral energy, $E_{\text {extra }}$, before performing the kinematic fit for the combined $\Upsilon(2 S)$ and $\Upsilon(3 S)$ datasets, together with various background estimates (stacked histograms) and signal MC predictions (solid line). The requirement on $E_{\text {extra }}$ is indicated by a dashed line. The signal MC prediction is normalized to a branching fraction $\mathcal{B}(\Upsilon \rightarrow S \bar{\Lambda} \bar{\Lambda})=5 \times 10^{-7}$. The background MC normalization is described in the text. 


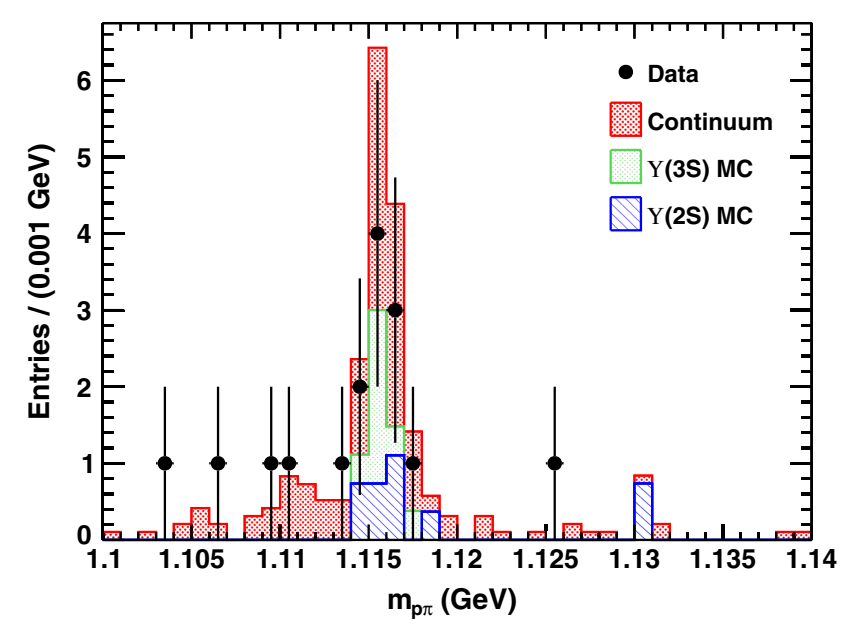

FIG. 2. The distribution of the $p \pi$ invariant mass, $m(p \pi)$, before performing the kinematic fit for the combined $\Upsilon(2 S)$ and $\Upsilon(3 S)$ datasets, together with various background estimates (stacked histograms). Two entries per event are plotted.

candidates, providing a better estimator of the efficiency near $m_{S} \sim 0 \mathrm{GeV}$ than the recoil mass. The $m_{\text {rec }}^{2}$ distribution is shown in Fig. 3(a), together with various background predictions and a simulated signal assuming $m_{S}=$ $1.6 \mathrm{GeV}$ and a branching fraction $\mathcal{B}(\Upsilon \rightarrow S \bar{\Lambda} \bar{\Lambda})=$ $1 \times 10^{-7}$. No events are observed in the signal region, and the expected background is found to be negligible as well.

The continuum $e^{+} e^{-} \rightarrow q \bar{q}(q=u, d, s, c)$ background is estimated from the data collected at the $\Upsilon(4 S)$ peak. This data sample contains contributions from both continuum and $\Upsilon(4 S)$ events. The latter is evaluated from the generic $\Upsilon(4 S)$ MC sample and found to be negligible, as those decays tend to have higher multiplicity and are much more suppressed than continuum production by our selection. The data collected at the $\Upsilon(4 S)$ resonance are therefore a good representation of the continuum background.

The $\Upsilon(2 S, 3 S)$ background components are estimated from the corresponding MC simulations. The contributions are normalized using sideband data obtained by applying all the selection criteria previously described but requiring $E_{\text {extra }}$ to be greater than $0.5 \mathrm{GeV}$ instead of below that threshold. The $\Upsilon(2 S, 3 S)$ MC components are found to underestimate the observed $p \pi$ yield, and we adjust their overall normalizations to improve the agreement with the data. The resulting correction factors, determined to be respectively 1.25 and 1.55 for the $\Upsilon(2 S)$ and $\Upsilon(3 S)$ samples, are propagated throughout the analysis.

A data-driven estimate of the background is also derived from the sideband data. Similarly to signal events, this sample contains predominantly two real $\Lambda$ particles with additional (undetected) particles. Since the difference in $E_{\text {extra }}$ is essentially due to the interaction of those particles with the calorimeter, sideband data provide a good approximation of the expected background in the signal region.
The corresponding recoil mass distribution is displayed in Fig. 3(b). Similarly to the background estimate previously described, sideband data predict a negligible level of background in the signal region.

The efficiency as a function of the $S$ mass is derived from the corresponding MC sample. For each mass hypothesis, we define a signal region in the $m_{\mathrm{rec}}^{2}$ distribution as the symmetric interval around the nominal $S$ mass containing $99 \%$ of the reconstructed $S$ candidates. Its typical size is of the order of $2.5 \mathrm{GeV}^{2}$. The efficiency varies between $7.2 \%$ near threshold to $8.2 \%$ near $m_{S}=2 \mathrm{GeV}$. It is mainly driven by the detector acceptance and the $\Lambda \rightarrow p \pi$ branching fraction.

The main uncertainties on the efficiency arise from the modeling of the $\Upsilon \rightarrow S \bar{\Lambda} \bar{\Lambda}$ angular distribution and the limited knowledge of the $S$-matter interactions. The former varies between $4 \%$ to $15 \%$, assessed by taking the difference between the predictions based on the simplified
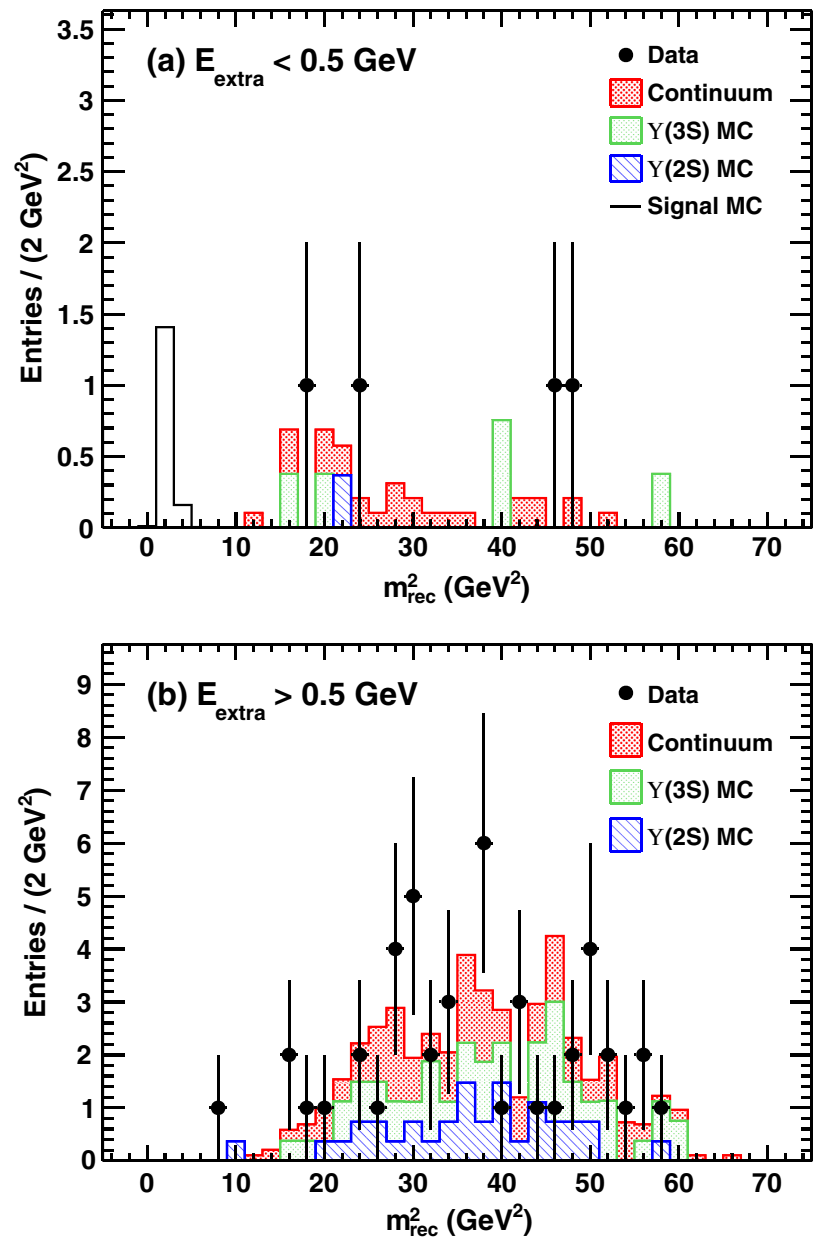

FIG. 3. The distribution of the recoil mass squared against the $\Lambda \Lambda$ system, $m_{\text {rec }}^{2}$, after performing the kinematic fit for the combined $\Upsilon(2 S)$ and $\Upsilon(3 S)$ datasets, together with various background estimates (stacked histograms) and a signal example for (a) the $E_{\text {extra }}<0.5 \mathrm{GeV}$ signal region and (b) the $E_{\text {extra }}>$ $0.5 \mathrm{GeV}$ sideband data sample. 


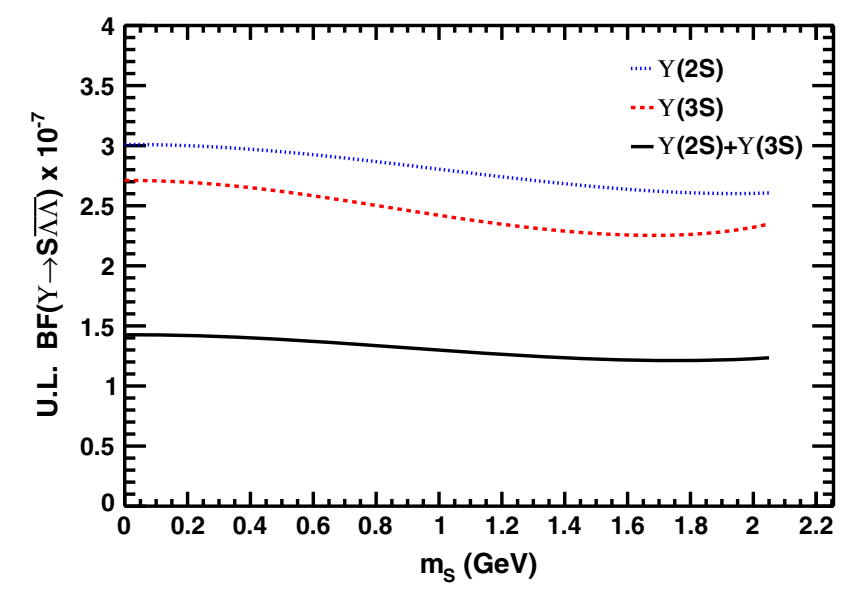

FIG. 4. The $90 \%$ C.L. upper limits on the $\Upsilon(2 S, 3 S) \rightarrow S \bar{\Lambda} \bar{\Lambda}$ branching fraction for the $\Upsilon(2 S)$ and $\Upsilon(3 S)$ datasets, as well as the combined sample assuming the same partial width.

Lagrangian to those obtained using a phase space distribution for $\Upsilon$ decays. The latter is estimated by considering the difference between simulations modeling the $S$ as a neutron or a non-interacting particle. The corresponding uncertainty ranges from $8 \%$ to $10 \%$. A systematic uncertainty of $8 \%$ is included to account for the difference in $\Lambda$ reconstruction efficiencies between data and MC calculations, determined from control samples in data [28]. Both the uncertainty on the $\Lambda \rightarrow p \pi$ branching fraction (1.6\% [29]) and the finite MC sample size $(\sim 1.5 \%)$ are also propagated.

No significant signal is observed, and we derive 90\% confidence level (C.L.) upper limits on the $\Upsilon(2 S, 3 S) \rightarrow S \bar{\Lambda} \bar{\Lambda}$ branching fractions, scanning $S$ masses in the range $0 \mathrm{GeV}<m_{S}<2.05 \mathrm{GeV}$ in steps of $50 \mathrm{MeV}$ (approximately half the signal resolution). For each mass hypothesis, we evaluate the upper bound on the number of signal events from the $m_{\mathrm{rec}}^{2}$ distribution with a profile likelihood method [30]. This approach treats the background as a Poisson process whose unknown mean is estimated from the number of observed background events, set to zero in this instance. Systematic uncertainties are included by modeling the signal efficiency as a Gaussian distribution with the appropriate variance. In addition to the contributions previously described, the limits include an additional uncertainty of $0.6 \%$ associated with the uncertainty on the number of $\Upsilon(2 S)$ and $\Upsilon(3 S)$ decays. The results are shown in Fig. 4 for the $\Upsilon(2 S)$ and $\Upsilon(3 S)$ datasets, as well as the combined sample assuming the same partial width.

In conclusion, we performed the first search for a stable uuddss configuration in $\Upsilon$ decays. No signal is observed, and $90 \%$ C.L. limits on the combined $\Upsilon(2 S, 3 S) \rightarrow S \bar{\Lambda} \bar{\Lambda}$ branching fraction of $(1.2-1.4) \times 10^{-7}$ are derived for $m_{S}<2.05 \mathrm{GeV}$. These results set stringent bounds on the existence of a stable, doubly strange six-quark state.

We thank G. Farrar for providing us with the theoretical model to simulate the signal and useful discussions. We are grateful for the extraordinary contributions of our PEP-II2 colleagues in achieving the excellent luminosity and machine conditions that have made this work possible. The success of this project also relies critically on the expertise and dedication of the computing organizations that support $B A B A R$. The collaborating institutions wish to thank SLAC for its support and the kind hospitality extended to them. This work is supported by the US Department of Energy and National Science Foundation, the Natural Sciences and Engineering Research Council (Canada), the Commissariat à l'Energie Atomique and Institut National de Physique Nucléaire et de Physique des Particules (France), the Bundesministerium für Bildung und Forschung and Deutsche Forschungsgemeinschaft (Germany), the Istituto Nazionale di Fisica Nucleare (Italy), the Foundation for Fundamental Research on Matter (Netherlands), the Research Council of Norway, the Ministry of Education and Science of the Russian Federation, Ministerio de Economía y Competitividad (Spain), the Science and Technology Facilities Council (United Kingdom), and the Binational Science Foundation (U.S.-Israel). Individuals have received support from the Marie-Curie IEF program (European Union) and the A. P. Sloan Foundation (USA).

Appendix.-The $S$ angular distribution is simulated using the following amplitude:

$$
\begin{aligned}
|A|^{2}= & 2 \frac{m M\left(m^{2}-\alpha\right)-M^{2} \beta+2(m M+\alpha-\beta)(\beta-\gamma-m M)-m M\left(\gamma-m^{2}\right)+m^{2}\left(\alpha-\beta+\gamma-M_{Y}^{2}\right)}{\left(m^{2}-M^{2}-2 \alpha+M_{Y}^{2}\right)\left(m^{2}-M^{2}-2 \gamma+M_{Y}^{2}\right)} \\
& +\frac{2 m^{2}\left(M^{2}+m^{2}-2 \alpha+M_{Y}^{2}\right)-2 m M\left(m^{2}-\alpha-\beta+\gamma\right)+2\left(m^{2}-\alpha\right)(\beta-\gamma)-\beta\left(M_{Y}^{2}+m^{2}-M^{2}-2 \alpha\right)}{\left(m^{2}-M^{2}-2 \alpha+M_{Y}^{2}\right)^{2}} \\
& +\frac{2 m^{2}\left(M^{2}-m^{2}-2 \gamma+M_{Y}^{2}\right)+2 m M\left(\alpha-\beta+\gamma-m^{2}\right)-2\left(m^{2}-\gamma\right)(\alpha-\beta)-\beta\left(M_{Y}^{2}-m^{2}-M^{2}-2 \gamma\right)}{\left(m^{2}-M^{2}-2 \gamma+M_{Y}^{2}\right)^{2}}
\end{aligned}
$$


where $\alpha=p \cdot q, \beta=p \cdot p^{\prime}, \gamma=p^{\prime} \cdot q, q$ is the 4-momentum of the $\Upsilon(2 S, 3 S), p\left(p^{\prime}\right)$ is the 4-momentum of the first (second) $\Lambda, m$ is the $\Lambda$ mass and $M$ is an effective mass, taken to be $m_{\Lambda}$.

*Deceased.

Present address: Wuhan University, Wuhan 430072, China. ${ }^{\star}$ Present address: Università di Bologna and INFN Sezione di Bologna, I-47921 Rimini, Italy.

${ }^{\S}$ Present address: University of Huddersfield, Huddersfield HD1 3DH, United Kingdom.

"Present address: University of South Alabama, Mobile, Alabama 36688, USA.

*** Also at Università di Sassari, I-07100 Sassari, Italy.

${ }^{\dagger \dagger}$ Also at Gran Sasso Science Institute, I-67100 L'Aquila, Italy.

[1] J. Preskill, Nucl. Phys. B177, 21 (1981).

[2] R. L. Jaffe, Phys. Rev. Lett. 38, 195 (1977); 38, 617(E) (1977).

[3] Natural units $(\hbar=c=1)$ are used throughout this Letter.

[4] J. K. Ahn et al., Phys. Rev. Lett. 87, 132504 (2001).

[5] H. Takahashi et al., Phys. Rev. Lett. 87, 212502 (2001).

[6] B. H. Kim et al. (Belle Collaboration), Phys. Rev. Lett. 110, 222002 (2013).

[7] J. Badier et al. (NA3 Collaboration), Z. Phys. C 31, 21 (1986).

[8] R. H. Bernstein, T. K. Shea, B. Winstein, R. D. Cousins, Jr., J. F. Greenhalgh, M. Schwartz, G. J. Bock, D. Hedin, and G. B. Thomson, Phys. Rev. D 37, 3103 (1988).

[9] J. Belz et al. (BNL-E888 Collaboration), Phys. Rev. Lett. 76, 3277 (1996); Phys. Rev. C 56, 1164(A) (1997).

[10] A. Alavi-Harati et al. (KTeV Collaboration), Phys. Rev. Lett. 84, 2593 (2000).
[11] H. R. Gustafson, C. A. Ayre, L. W. Jones, M. J. Longo, and P. V. Ramana Murthy, Phys. Rev. Lett. 37, 474 (1976).

[12] G. R. Farrar, arXiv:1708.08951.

[13] Referred to as sexaquark by Farrar in Ref. [12].

[14] S. R. Beane, E. Chang, S. D. Cohen, W. Detmold, H. W. Lin, T. C. Luu, K. Orginos, A. Parreño, M. J. Savage, and A. Walker-Loud (NPLQCD Collaboration), Phys. Rev. D 87, 034506 (2013).

[15] A. Francis, J. R. Green, P. M. Junnarkar, C. Miao, T. D. Rae, and H. Wittig, arXiv:1805.03966 and references therein.

[16] C. Gross, A. Polosa, A. Strumia, A. Urbano, and W. Xue, Phys. Rev. D 98, 063005 (2018).

[17] E. W. Kolb and M. S. Turner, arXiv:1809.06003.

[18] S. D. McDermott, S. Reddy, and S. Sen, arXiv:1809.06765.

[19] G. R. Farrar, arXiv:1805.03723.

[20] Charge conjugation is implied throughout the text.

[21] The search is also applicable to any scenario with a longlived six-quark state escaping the detector.

[22] J. P. Lees et al. (BABAR Collaboration), Nucl. Instrum. Methods Phys. Res., Sect. A 726, 203 (2013).

[23] B. Aubert et al. (BABAR Collaboration), Nucl. Instrum. Methods Phys. Res., Sect. A 479, 1 (2002).

[24] B. Aubert et al. (BABAR Collaboration), Nucl. Instrum. Methods Phys. Res., Sect. A 729, 615 (2013).

[25] G. R. Farrar (private communication).

[26] D. J. Lange, Nucl. Instrum. Methods Phys. Res., Sect. A 462, 152 (2001).

[27] S. Agostinelli et al. (GEANT4 Collaboration), Nucl. Instrum. Methods Phys. Res., Sect. A 506, 250 (2003).

[28] B. Aubert et al. (BABAR Collaboration), Phys. Rev. D 76, 092006 (2007).

[29] M. Tanabashi et al. (Particle Data Group), Phys. Rev. D 98, 030001 (2018).

[30] W. A. Rolke, A. M. Lopez, and J. Conrad, Nucl. Instrum. Methods Phys. Res., Sect. A 551, 493 (2005). 